\title{
Statics and dynamics of a self-bound matter-wave quantum ball
}

\author{
S. K. Adhikari*1 \\ ${ }^{1}$ Instituto de Física Teórica, UNESP - Universidade Estadual Paulista, 01.140-070 São Paulo, São Paulo, Brazil
}

\begin{abstract}
We study the statics and dynamics of a stable, mobile, three-dimensional matter-wave spherical quantum ball created in the presence of an attractive two-body and a very small repulsive threebody interaction. The quantum ball can propagate with a constant velocity in any direction in free space and its stability under a small perturbation is established numerically and variationally. In frontal head-on and angular collisions at large velocities two quantum balls behave like quantum solitons. Such collision is found to be quasi elastic and the quantum balls emerge after collision without any change of direction of motion and velocity and with practically no deformation in shape. When reflected by a hard impenetrable plane, the quantum ball bounces off like a wave obeying the law of reflection without any change of shape or speed. However, in a collision at small velocities two quantum balls coalesce to form a larger ball which we call a quantum-ball breather. We point out the similarity and difference between the collision of two quantum and classical balls. The present study is based on an analytic variational approximation and a full numerical solution of the mean-field Gross-Pitaevskii equation using the parameters of ${ }^{7} \mathrm{Li}$ atoms.
\end{abstract}

PACS numbers: 03.75.Lm, 03.75.Kk, 03.75.Nt

\section{INTRODUCTION}

After the experimental observation of Bose-Einstein condensate (BEC) [1, 2], there have been many experimental studies to investigate different quantum phenomena in a laboratory previously not accessible for study in a controlled environment, such as, quantum phase transition [3, quantum collapse of matter wave under attraction [4, four-wave mixing of matter waves [5], formation of vortex lattice [6, interference of matter waves [7, Josephson tunneling [], Anderson localization [9] etc. The generation and the dynamics of self-bound large quantum waves have drawn much attention lately. There have been some studies of self-bound matter waves or solitons in one (1D) 10 or two (2D) 11 space dimensions. A (self-bound) bright soliton travels at a constant velocity in $1 \mathrm{D}$, due to a cancellation of nonlinear attraction and defocusing forces [12, 13. The collision between two analytic 1D bright solitons is always elastic [12] with the solitons emerging after collision without a change of velocity and shape. The 1D soliton has been observed in nonlinear optics [12] and in Bose-Einstein condensates [10. However, a two- or three-dimensional (3D) soliton cannot be realized for two-body contact attraction alone due to collapse [12. The 1D BEC solitons studied experimentally [10] are really quasi solitons behaving like real solitons at large velocities. At low velocities a deformation of their shapes is expected upon collision.

There have been a few proposals for creating a selfbound 3D matter-wave state which we term a quantum ball. Some of these proposals involve an engineering of the atomic scattering length using the Feshbach resonance technique to generate dynamically stabilized solitons in 2D and 3D [14. Others consider extra inter-

\footnotetext{
*adhikari@ift.unesp.br; URL:http://www.ift.unesp.br/users/adhikari
}

actions usually neglected in dilute BEC of alkali atoms to create a stationary localized state. In the presence of an additional nonlocal dipolar interaction a 2D BEC soliton can be generated [11. It has been suggested by Maucher et al. 15 that in the case of Rydberg atoms, off-resonant dressing to Rydberg $\mathrm{nD}$ states can provide a nonlocal long-range attraction which can form a quantum ball. The collapse instability can be stopped in this case by a repulsive contact interaction.

In this paper we demonstrate that a very small repulsive three-body interaction in the presence of an attractive two-body contact interaction can generate a stable quantum ball. The collapse is stopped in this case by the repulsive three-body interaction. Although, some theoretical suggestions for generating a quantum ball, in the presence of a repulsive core in the two-body atomic interaction, seem viable [15, 16], so far there has not been success in their experimental realization. Nevertheless, there are questions about the dynamics of a quantum ball which are very intriguing. The study of the dynamics of a quantum elementary particle like an electron to determine simultaneously its position and velocity is doomed to failure due to the Heisenberg uncertainty relation. On the other hand, for a quantum ball the uncertainty relation is not of concern due to its large mass and it can be traced like a classical object by its position and velocity at each instant. As the quantum ball is self bound it can move like a classical ball obeying Newton's first law of motion. However, very little is known about the interaction dynamics of two quantum balls and that of a quantum ball with other objects and we address these questions in this paper.

We consider the mean-field Gross-Pitaevskii (GP) equation with the inclusion of a three-body interaction for the study of statics and dynamics of a quantum ball. The quantum balls are bound by an attractive two-body contact interaction in the presence of a repulsive threebody contact interaction. We use the realistic param- 
eters of ${ }^{7} \mathrm{Li}$ atoms, in this study, with a negative scattering length corresponding to two-body attraction [2]. The effect of atom loss due to three-body recombination is included in the study of dynamics. The three body loss rate is not accurately known for this system [17] for the parameter domain used in this study. We have chosen three-body loss rates that ensure our system does not decay significantly during our dynamical simulations. It is expected that the dynamics of quantum balls will be independent of the details of the mechanism responsible for self binding and we do not believe that the results obtained here are so peculiar as to have no general validity. In fact, a preliminary study revealed similar dynamics for quantum balls made of dipolar atoms [18. A stationary quantum ball can be formed for the two-body attraction above a critical value in the 3D GP equation for any finite three-body repulsion. The statical properties of the quantum ball are studied using a variational analysis and a numerical solution of the 3D GP equation. The variational and numerical results are found to be in good agreement with each other. The stability of the quantum ball is established numerically under a small perturbation introduced by changing the three-body interaction by a small amount, while the quantum ball is found to execute sustained breathing oscillation.

A quantum ball can move freely without deformation along any direction with a constant velocity. We study the frontal and angular collisions between two quantum balls. Only the collision between two integrable 1D solitons is truly elastic 12. As the dimensionality of the soliton is increased such collision is expected to become inelastic with loss of energy in $2 \mathrm{D}$ and $3 \mathrm{D}$. In the present numerical simulation of frontal collision between two quantum balls, at sufficiently large velocities the collision is found to be quasi elastic when the two quantum balls emerge after collision with practically no deformation and without any change of velocities. Unlike classical balls, obeying the Newton's laws, there is no change in the directions of motion of the quantum balls after collision. However, upon impact with a rigid impenetrable plane the quantum ball bounces like a classical elastic ball obeying the usual laws of reflection. At small velocities the collision between two quantum balls is inelastic and the quantum balls form a single bound entity in an excited state executing breathing oscillation, which we call a quantum-ball breather.

We present the 3D GP equation used in this study in Sec. II and a variational analysis of the same for an analytic understanding of the formation of the quantum ball. In Sec. III we present the numerical results for stationary profiles of a quantum ball. We present numerical tests of stability of a quantum ball under a small perturbation. The quasi-elastic nature of collision of two quantum balls at large velocities and the formation of a quantum-ball breather at small velocities are demonstrated by realtime simulation. We end with a summary of our findings in Sec. IV.

\section{MEAN-FIELD MODEL}

We consider a quantum ball in the presence of a threebody interaction and the mean-field model appropriate for this study. The mean-field GP equation for $N$ atoms of mass $m$ is 2 ]

$$
\begin{aligned}
i \hbar \frac{\partial \phi(\mathbf{r}, t)}{\partial t}= & {\left[-\frac{\hbar^{2}}{2 m} \nabla^{2}-\frac{4 \pi \hbar^{2}|a| N}{m}|\phi|^{2}\right.} \\
& \left.+\frac{\hbar N^{2} K_{3}}{2}|\phi|^{4}\right] \phi(\mathbf{r}, t),
\end{aligned}
$$

where $a$ is the scattering length, and $K_{3}$ is the threebody interaction term. The negative scattering length $a$ represents two-body attraction and the positive $K_{3}$ to three-body repulsion.

For an analytic understanding of the formation of a quantum ball convenient variational approximation of Eq. (1) can be obtained with the following Gaussian ansatz for the time-independent stationary wave function [19.

$$
\phi(\mathbf{r})=\frac{\pi^{-3 / 4}}{w^{3 / 2}} \exp \left[-\frac{r^{2}}{2 w^{2}}\right],
$$

where $r^{2}=x^{2}+y^{2}+z^{2}, w$ is the width. The energy density corresponding to Eq. (1) is given by

$\mathcal{E}(\mathbf{r})=\frac{\hbar^{2}|\nabla \phi(\mathbf{r})|^{2}}{2 m}-\frac{2 \pi N|a| \hbar^{2}|\phi(\mathbf{r})|^{4}}{m}+\frac{\hbar N^{2} K_{3}|\phi(\mathbf{r})|^{6}}{6}$.(3)

Consequently, the total energy per atom $E \equiv \int \mathcal{E}(\mathbf{r}) d \mathbf{r}$ becomes

$$
E=\frac{\hbar^{2}}{m} \frac{3}{4 w^{2}}-\frac{4 \pi N|a| \hbar^{2}}{m} \frac{\pi^{-3 / 2}}{4 \sqrt{2} w^{3}}+\frac{\hbar N^{2} K_{3}}{2} \frac{\pi^{-3}}{9 \sqrt{3} w^{6}} .
$$

The width $w$ of a stationary quantum ball with negative energy corresponds to a global minimum of energy $E$ :

$$
\frac{1}{w^{3}}-\frac{4 \pi N|a|}{(2 \pi)^{3 / 2} w^{4}}+\frac{m N^{2} K_{3}}{2 \hbar} \frac{4 \pi^{-3}}{9 \sqrt{3} w^{7}}=0 .
$$

Without the quintic term $\left(K_{3}=0\right)$ the quantum ball of width $w=4 \pi N|a| /(2 \pi)^{3 / 2}$ is tantamount to an unstable Towne's soliton [20]. For stability a non-zero quintic term $\left(K_{3}>0\right)$ is necessary.

\section{NUMERICAL RESULTS}

In the numerical calculation, we use the parameters of ${ }^{7} \mathrm{Li}$ atoms, e.g., $a \approx-27.4 a_{0}[2,21$ and $m=7 \mathrm{amu}$, where $a_{0}$ is the Bohr radius. Unlike the $1 \mathrm{D}$ case, the 3D GP equation (1) does not have analytic solution and different numerical methods, such as split-step CrankNicolson [22] and Fourier spectral 23] methods, are used for its solution. We solve the 3D GP equation (1) numerically by the split-step Crank-Nicolson method using both 

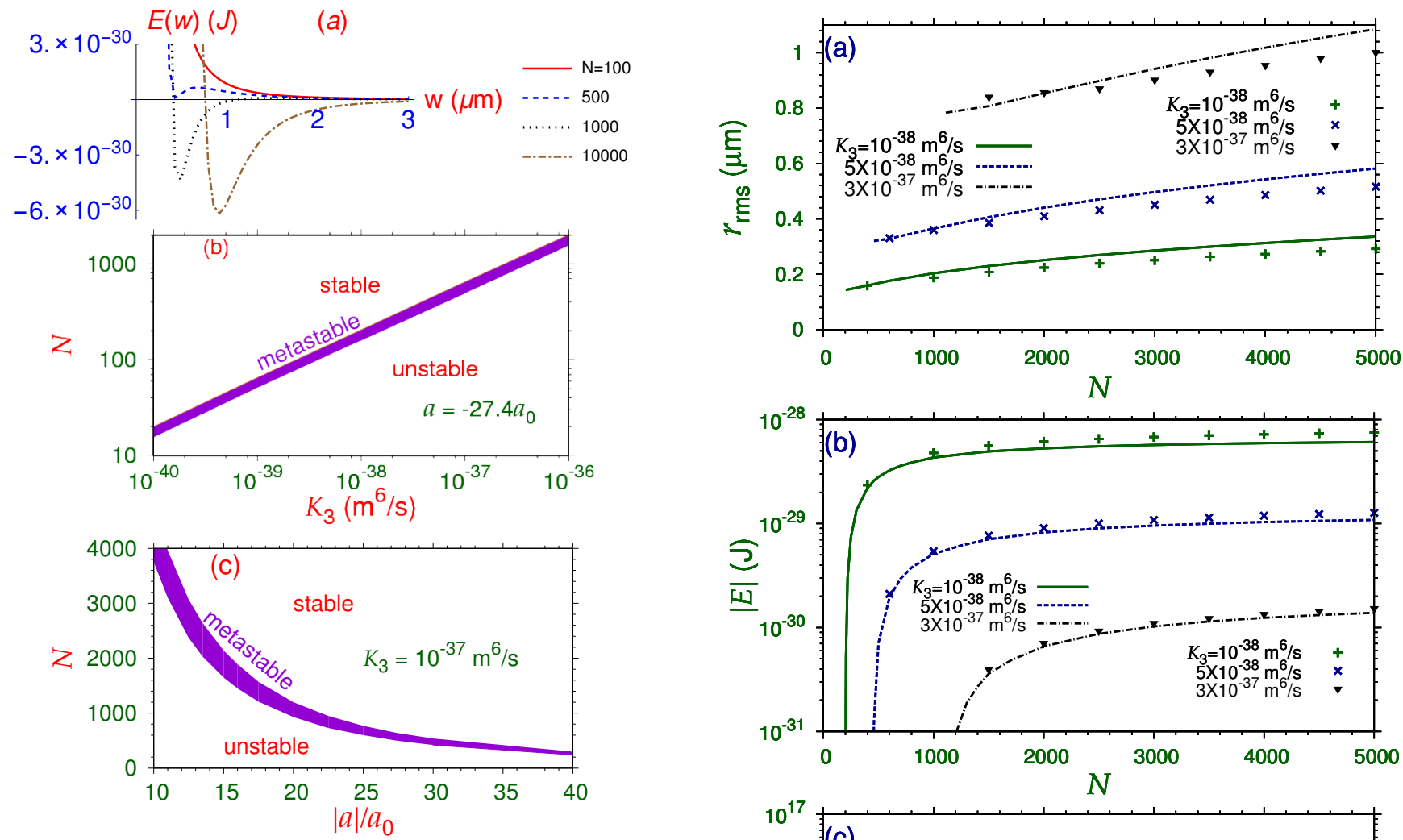

FIG. 1: (Color online) (a) The variational energy $E$ versus $w$ in $\mu \mathrm{m}$ for different $N$ from Eq. (5) for $a=-27.4 a_{0}$ and $K_{3}=$ $10^{-37} \mathrm{~m}^{6} / \mathrm{s}$. Variational (b) $N-K_{3}$ and (c) $N-a / a_{0}$ phase plots for $a=-27.4 a_{0}$ and $K_{3}=10^{-37} \mathrm{~m}^{6} / \mathrm{s}$, respectively, illustrating the regions of formation of a stable and metastable quantum ball obtained from Eq. (5).

real- and imaginary-time propagation in Cartesian coordinates using a space step of $0.025 \mu \mathrm{m}$ and a time step of $0.00002 \mathrm{~ms}$ in all calculations [22]. All imaginary-time simulations were performed in a box of size $240 \times 240 \times 240$ unless otherwise stated. Imaginary-time simulation is employed to get the lowest-energy bound state of a quantum ball, while the real-time simulation is to be used to study the dynamics using the initial profile obtained in the imaginary-time propagation 22. There are different $\mathrm{C}$ and FORTRAN programs for solving the GP equation [22, 24] and one should use the appropriate one. In the imaginary-time propagation the initial state was taken as in Eq. (1) and the width $w$ set equal to the variational solution obtained by solving Eq. (5). The convergence will be quick if the guess for the width $w$ is close to the final width.

The variational width of a stationary quantum ball can be obtained from a solution of Eq. (5). If a minimum of energy (4) exists, it can be either a global minimum with negative energy corresponding to a stable state or a local minimum of positive energy corresponding to a metastable state. The energy $E \rightarrow \infty$ at $w=0$ even for

a very small non-zero $K_{3}$, and $E=0$ as $w \rightarrow \infty$. Hence collapse is not allowed in the presence of a very small three-body repulsion. For certain values of the parameters there is a negative energy region between these two limiting values where the global minimum corresponding to a stable stationary state is located. For other sets of parameters, the energy changes monotonically between the above two limiting values without ever becoming negative or may have a local minimum with positive energy corresponding to a metastable state. This is illustrated in Fig. 1(a) for ${ }^{7} \mathrm{Li}$ atoms by a plot of $E$ versus $w$ for different $N$ from Eq. (4) for $a=-27.4 a_{0}$ and $K_{3}=10^{-37}$ 


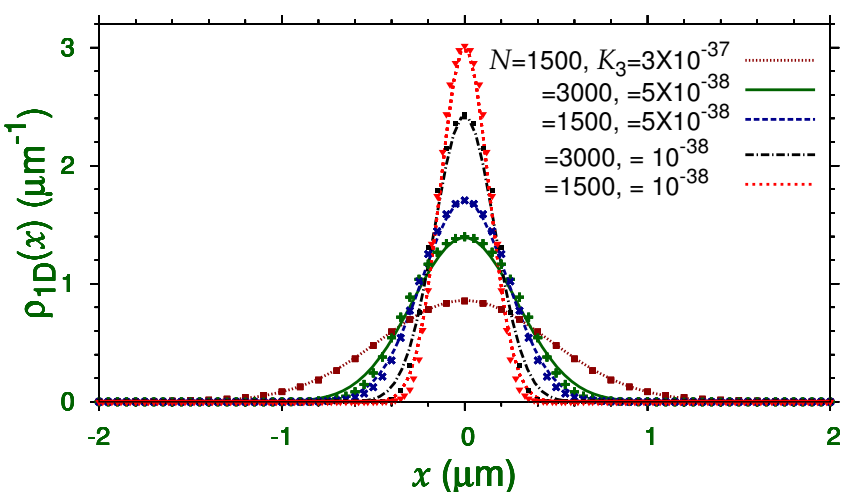

FIG. 3: (Color online) Numerical (chain of symbols) and variational (line) reduced $1 \mathrm{D}$ density $\rho_{1 D}(x)$ of a ${ }^{7} \mathrm{Li}$ quantum ball for different $N$ and $K_{3}$.

$\mathrm{m}^{6} / \mathrm{s}$. For $N=100$ there is no minimum of $E$ and there cannot be a quantum ball. For $N=500$ there is a minimum at positive energy corresponding to a metastable state. Finally, for $N=1000$ and 10000 there are minima at negative energies corresponding to stable states. The parameter domains for the formation of stable and metastable states are shown in $N-K_{3}$ and $N-|a| / a_{0}$ phase plots for $a=-27.4 a_{0}$ and $K_{3}=10^{-37} \mathrm{~m}^{6} / \mathrm{s}$, respectively, in Figs. 1.(b) and (c). An interesting scaling relation $N \sim \sqrt{K_{3}}$ is noted in Fig. 11(b). Although there is a lower limit on the number of atoms $N$ for the formation of a stable quantum ball, viz. Figs. 1(b) and (c), there is no upper limit on $N$. In the following we will only be concerned with the global minimum with negative energy corresponding to a stable stationary state.

Next we compare in Fig. 2(a) the numerical and variational root-mean-square (rms) radius $r_{\text {rms }}$ of a ${ }^{7} \mathrm{Li}$ quantum ball versus number of atoms $N$ for three different values of the three-body term: $K_{3}=10^{-38} \mathrm{~m}^{6} / \mathrm{s}$, $=5 \times 10^{-38} \mathrm{~m}^{6} / \mathrm{s},=3 \times 10^{-37} \mathrm{~m}^{6} / \mathrm{s}$. The variational result for the rms radius is given by: $r_{\mathrm{rms}}=\sqrt{3 / 2} w$, where $w$ is the equilibrium variational width. For small $N$, the quantum balls are well localized with small size and the agreement between numerical and variational results is better. In Fig. 2(b) we show the numerical and variational energies $|E|$ of a quantum ball versus $N$ for different $K_{3}$. The energy of a bound quantum ball is negative in all cases and its absolute value is plotted. In Fig. 2(c) the numerical and variational peak atom density $n \equiv N|\phi(0,0,0)|^{2}$ of the quantum ball versus $N$ is illustrated for different $K_{3}$.

To study the density distribution of a ${ }^{7} \mathrm{Li}$ quantum ball we calculate the reduced $1 \mathrm{D}$ density defined by

$$
\rho_{1 \mathrm{D}}(x)=\int d z d y|\phi(\mathbf{r})|^{2} .
$$

In Fig. 3 we plot this reduced 1D density as obtained from variational and numerical calculations for different $N$ and $K_{3}$. For a fixed three-body term $K_{3}$, the quantum ball is more compact with the decrease of number

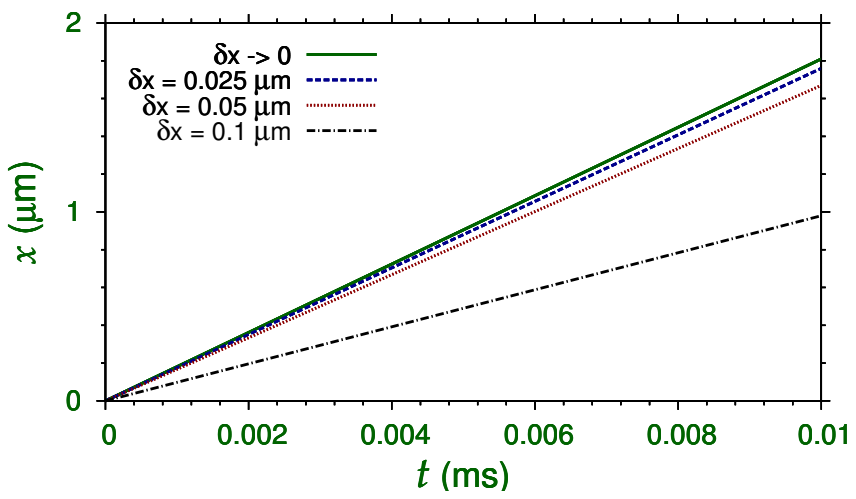

FIG. 4: (Color online)The displacement versus time for the quantum ball of $N=1500$ and $K_{3}=3 \times 10^{-37} \mathrm{~m}^{6} / \mathrm{s}$ moving with velocity $v=18.16 \mathrm{~cm} / \mathrm{s}$ calculated with different space steps $\delta x$.

of atoms $N$. For a fixed number of atoms $N$, the quantum ball is more compact for a small three-body term $K_{3}$. The agreement between the two densities is better for the compact quantum balls of smaller size as in Fig. 2.

We performed numerical tests of stability of the ${ }^{7} \mathrm{Li}$ quantum balls under a small perturbation (details not reported here). We considered a quantum-ball wave function as calculated by imaginary-time propagation and performed real-time propagation with the imaginarytime wave function under a small perturbation introduced at $t=0$ upon changing the scattering length by less than $1 \%$. After this sudden perturbation the quantum ball starts a breathing oscillation. The continued oscillation of the quantum ball over a long interval of time establishes its stability.

To study the dynamics of the quantum balls, we need to set these in motion. This can be achieved by multiplying the imaginary-time wave function by a phase $\exp ( \pm i p x / \hbar)$ with $p / \hbar \equiv m v / \hbar$, where $p$ and $v$ are momentum and velocity, and real-time simulation is then performed using these wave functions for the study of moving quantum balls with velocity $v$. However, to achieve the desired velocity numerically an accurate wave function calculated over a large space domain and small space and time steps are needed; otherwise the numerically generated velocity is always less than the expected velocity $v$, except when $v$ is very small. This is illustrated in Fig. 4 where we plot the displacement versus time for the moving quantum ball, along the $x$ axis, of $N=1500$ and $K_{3}=3 \times 10^{-37} \mathrm{~m}^{6} / \mathrm{s}$ after multiplying its wave function by $\exp (i 20 x)$, so that $p / \hbar \equiv m v / \hbar=20$ $\mu \mathrm{m}^{-1}=20 \times 10^{6} \mathrm{~m}^{-1}$ leading to $v=18.16 \mathrm{~cm} / \mathrm{s}$. The numerically generated dynamics was obtained with space steps $\delta x=0.1 \mu \mathrm{m}, 0.05 \mu \mathrm{m}$, and $0.025 \mu \mathrm{m}$ and illustrated in Fig. 4. The ideal theoretical result for $\delta x \rightarrow 0$ is also shown. The result for $\delta x=0.025 \mu \mathrm{m}$ is satisfactory and this value of space step has been used in all calculations. 

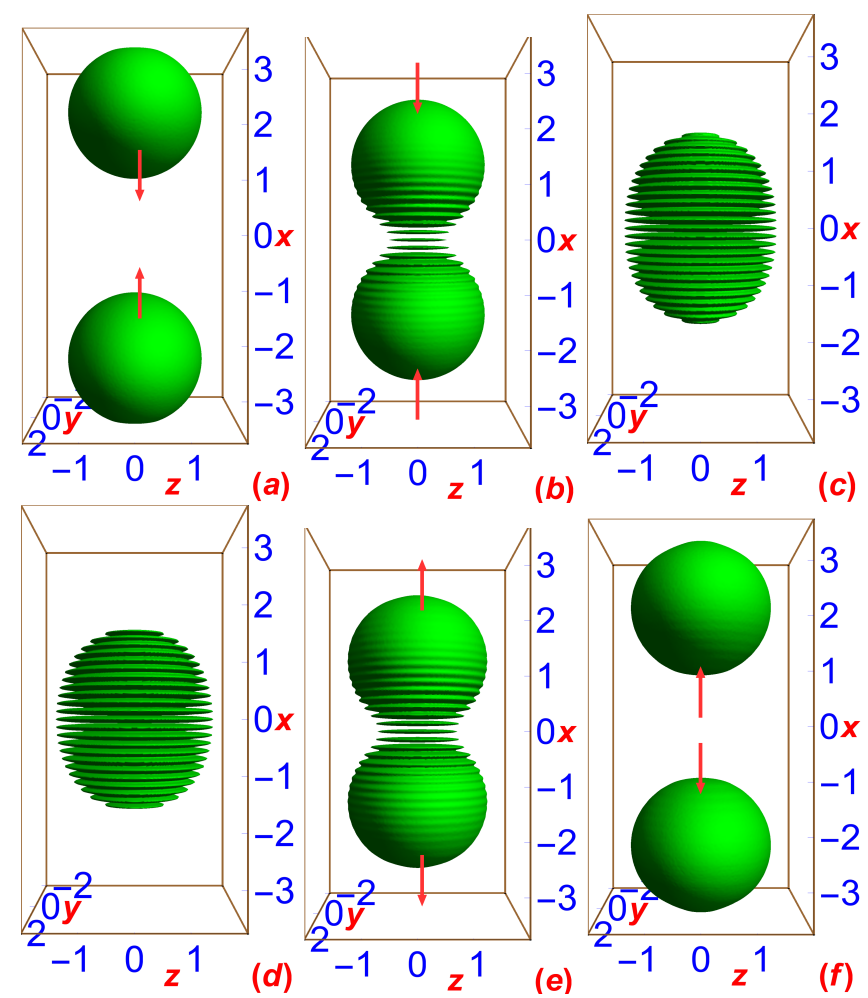

(c)
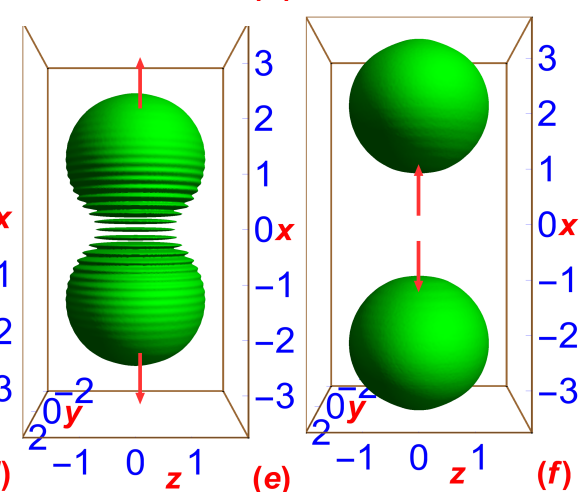

FIG. 5: (Color online) Collision dynamics of two ${ }^{7} \mathrm{Li}$ quantum balls, with $N=1500, K_{3}=3 \times 10^{-37}(1-i) \mathrm{m}^{6} / \mathrm{s}$ each, placed at $x= \pm 2.5 \mu \mathrm{m}$ at $t=0 \mathrm{~ms}$ and set into motion in opposite directions along the $x$ axis with velocity $18.16 \mathrm{~cm} / \mathrm{s}$, so as to collide at $x=0$, illustrated by isodensity contours at times (a) $t=0,(\mathrm{~b})=0.0057 \mathrm{~ms},(\mathrm{c})=0.0114 \mathrm{~ms},(\mathrm{~d})$ $=0.017 \mathrm{~ms},(\mathrm{e})=0.0228 \mathrm{~ms},(\mathrm{f})=0.0285 \mathrm{~ms}$. The density on the contour is $10^{10}$ atoms $/ \mathrm{cm}^{3}$ and unit of length is $\mu \mathrm{m}$. The directions of motion of the quantum balls are shown by arrows.

In the following we study the collision dynamics of quantum balls, where we use a three-body term $K_{3}$ with dissipation corresponding to a loss of atoms from the quantum ball due to molecule formation. To test the solitonic nature of the present quantum balls, we study the frontal head-on collision of two quantum balls at large velocity including a three-body term with absorption: $K_{3}=3 \times 10^{-37}(1-i) \mathrm{m}^{6} / \mathrm{s}$. The imaginary-time profiles of the quantum balls shown in Fig. 3 with $N=1500$ and $K_{3}=3 \times 10^{-37} \mathrm{~m}^{6} / \mathrm{s}$ each are used as the initial wave functions in the real-time simulation of collision, with two identical quantum balls placed at $x= \pm 2.5 \mu \mathrm{m}$ at $t=0$. To set the quantum balls in motion along the $x$ axis in opposite directions the respective imaginarytime wave functions are multiplied by $\exp ( \pm i 20 x)$ and real-time simulation is then performed using these wave functions for the study of dynamics. The corresponding velocity is $v=18.16 \mathrm{~cm} / \mathrm{s}$. To illustrate the dynamics, we plot the isodensity contour of the colliding quantum balls in Fig. 5 at different times obtained by real-time simulation over a box of size $480 \times 240 \times 240$. The initial
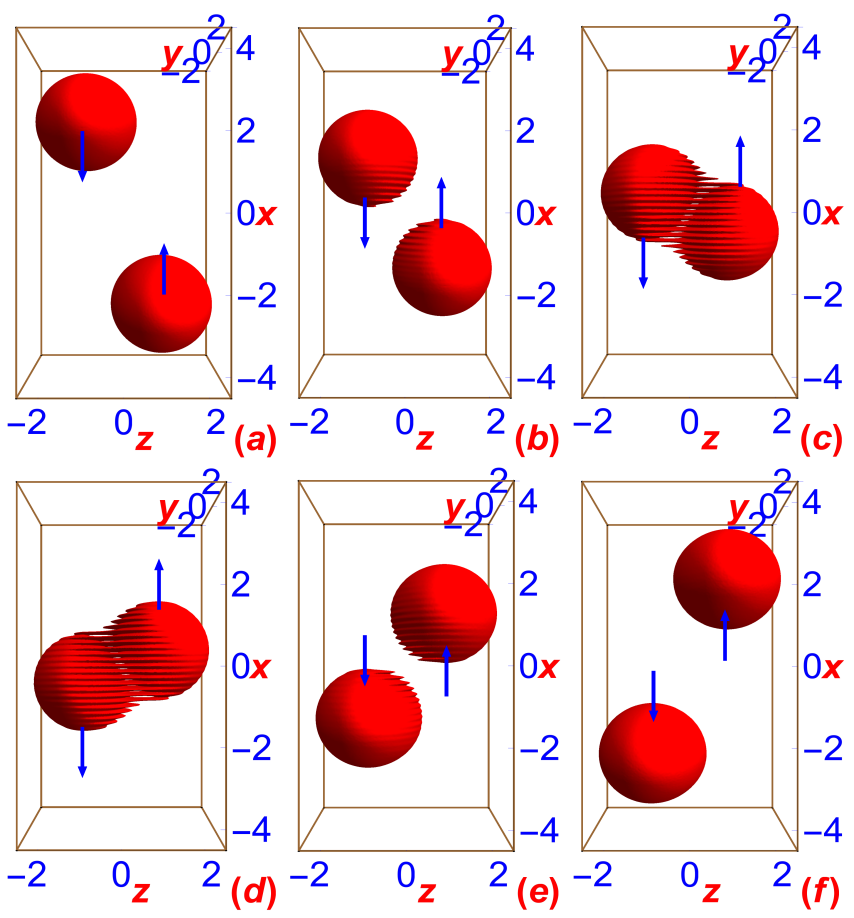

FIG. 6: (Color online) Collision dynamics of two ${ }^{7} \mathrm{Li}$ quantum balls, with $N=1500, K_{3}=3 \times 10^{-37}(1-i) \mathrm{m}^{6} / \mathrm{s}$ each, placed at $x= \pm 2.5 \mu \mathrm{m}, z=\mp 0.9 \mu \mathrm{m}$ at $t=0$ and set into motion in opposite directions along the $x$ axis with velocity $18.16 \mathrm{~cm} / \mathrm{s}$, so as to collide at $x=0$, illustrated by isodensity contours at times (a) $t=0,(\mathrm{~b})=0.0057 \mathrm{~ms}$, (c) $=0.0114 \mathrm{~ms}$, (d) $=0.017 \mathrm{~ms},(e)=0.0228 \mathrm{~ms},(\mathrm{f})=0.0285 \mathrm{~ms}$. The density on the contour is $10^{10}$ atoms $/ \mathrm{cm}^{3}$ and unit of length is $\mu \mathrm{m}$. The directions of motion of the quantum balls are shown by arrows.

profiles of the balls are shown in Fig. 5(a). The balls come close to each other in Fig. 5(b), coalesce to form a single entity in Figs. 5(c) and (d), form two separate balls in Fig. 5(e), and are well separated in Fig. 5 (f) with identical profiles as in Fig. 5(a). During collision, in Figs. 5 (c) and (d) the identity of the two separate balls give rise to a larger object which eventually breaks up into two quantum balls. Considering the three-dimensional nature of collision, the distortion in the profile of the quantum balls after collision is found to be negligible, recalling that their identities were lost during collision. It is useful to contrast the collision shown in Fig. 5 with the corresponding elastic frontal collision of two classical balls. In the quantum collision the identity of the balls is lost during interaction and a quantum ball cannot be followed during collision like a classical ball. Apart from that, the position and velocity of the quantum balls before and after collision are identical with those of the classical balls in this process.

Besides the frontal collision considered in Fig. 5 we also consider two types of nonfrontal collisions including a three-body term with absorption. First, we consider the collision between two quantum balls moving along 

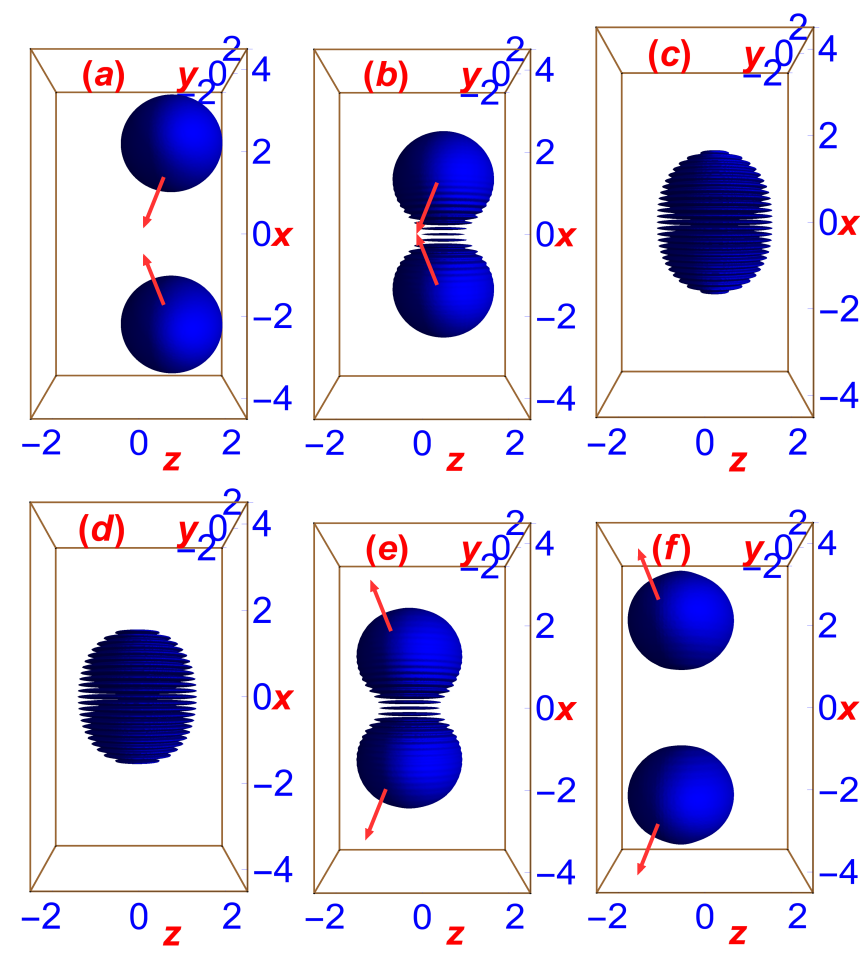

FIG. 7: (Color online) Collision dynamics of two ${ }^{7} \mathrm{Li}$ quantum balls, with $N=1500, K_{3}=3 \times 10^{-37}(1-i) \mathrm{m}^{6} / \mathrm{s}$ each, placed at $x= \pm 2.5 \mu \mathrm{m}, z=0.9 \mu \mathrm{m}$ at $t=0$ and set into motion towards origin $(x=z=0)$ with velocity $18.9 \mathrm{~cm} / \mathrm{s}$, illustrated by isodensity contours at times (a) $t=0$, (b) $=$ $0.0057 \mathrm{~ms},(\mathrm{c})=0.0114 \mathrm{~ms},(\mathrm{~d})=0.017 \mathrm{~ms},(\mathrm{e})=0.0228$ $\mathrm{ms}$, (f) $=0.0285 \mathrm{~ms}$. The density on the contour is $10^{10}$ atoms $/ \mathrm{cm}^{3}$ and unit of length is $\mu \mathrm{m}$. The directions of motion of the quantum balls are shown by arrows.

the $x$ axis but on laterally displaced tracks. At $t=0$ two balls of $N=1500$ and $K_{3}=3 \times 10^{-37}(1-i) \mathrm{m}^{6} / \mathrm{s}$ each are placed at $x= \pm 2.5 \mu \mathrm{m}, y=0, z=\mp 0.9 \mu \mathrm{m}$, respectively, and set into motion along $x$ axis in opposite directions by multiplying the respective imaginary-time wave functions by $\exp ( \pm i 20 x)$ and performing real-time simulation in a box of size $480 \times 240 \times 360$ with these wave functions for the study of dynamics. The collision is illustrated in Fig. 6(a)-(f) through successive snapshots of isodensity contours of the system before, during and after collision. In this case in Figs. 6(a) and (b) the balls approach along the $x$ axis, in Figs. 6(c) and (d) they interact by losing identity, and in Figs. 6(e) and (f) they eventually come out of the interaction region undeformed while moving along the $x$ axis maintaining their original trajectories and conserving their velocities. This collision has no classical analogue. In the elastic collision of two classical balls in this case, the balls will be deflected from their original trajectories conserving energy and momentum.

Another type of collision of interest is the angular collision of two quantum balls which we now study. For

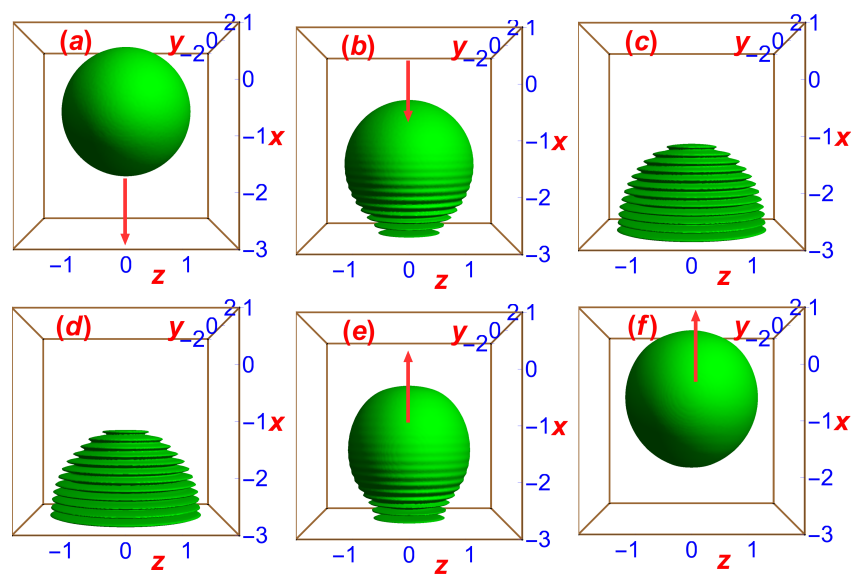

FIG. 8: (Color online) Bouncing off a rigid wall at $x=-0.5$ $\mu \mathrm{m}$ of a ${ }^{7} \mathrm{Li}$ quantum ball, with $N=1500, K_{3}=3 \times 10^{-37}(1-$ i) $\mathrm{m}^{6} / \mathrm{s}$, placed at origin at $t=0$ and moving along $x$ axis with the velocity of $18.18 \mathrm{~cm} / \mathrm{s}$, illustrated by isodensity contours at times (a) $t=0,(\mathrm{~b})=0.0057 \mathrm{~ms},(\mathrm{c})=0.0114 \mathrm{~ms},(\mathrm{~d})$ $=0.017 \mathrm{~ms},(\mathrm{e})=0.0228 \mathrm{~ms},(\mathrm{f})=0.0285 \mathrm{~ms}$. The density on the contour is $10^{10}$ atoms/cc and unit of length is $\mu \mathrm{m}$. The directions of motion of the quantum ball are shown by an arrows.

this purpose, at $t=0$ two balls of $N=1500$ and $K_{3}=3 \times 10^{-37}(1-i) \mathrm{m}^{6} / \mathrm{s}$ each are placed at $x= \pm 2.5$ $\mu \mathrm{m}, y=0, z=0.9 \mu \mathrm{m}$, respectively, and set into motion towards the origin $x=y=z=0$ with equal velocities by multiplying the respective imaginary-time wave functions by $\exp ( \pm i 20 x+i 5.8 z)$ to set the quantum balls in motion with a velocity of $18.9 \mathrm{~cm} / \mathrm{s}$ and performing realtime simulation in a box of size $480 \times 240 \times 360$ with these wave functions for the study of dynamics. Again the isodensity profiles of the quantum balls before, during, and after collision are shown in Figs. $7(\mathrm{a})-(\mathrm{b}),(\mathrm{c})-(\mathrm{d})$, and (e)-(f), respectively. In this case the balls again come out after collision undeformed maintaining their original trajectories and conserving their velocities. If we contrast this collision with the corresponding elastic collision of two classical balls, the position and velocity of the quantum balls before and after collision are identical with those of the classical balls in this process. However, again the quantum balls lose their identity during the collision.

The elastic interaction of a quantum ball with external objects is also of interest. For this purpose we consider its interaction with a rigid elastic plane upon perpendicular and angular impacts including a three-body term with absorption. To study the vertical impact with a rigid elastic plane we place a quantum ball of $N=1500$ and $K_{3}=3 \times 10^{-37}(1-i) \mathrm{m}^{6} / \mathrm{s}$ at $x=-0.5$, and $\mathrm{Q} y=z=0$ at $t=0$ and set it in motion along the $x$ axis with a velocity of $18.18 \mathrm{~cm} / \mathrm{s}$ by multiplying the imaginarytime wave function by $\exp (-i 20 x)$ and performing realtime simulation with this wave function in a box of size $240 \times 240 \times 240$. The reflection of the ball from the wall 

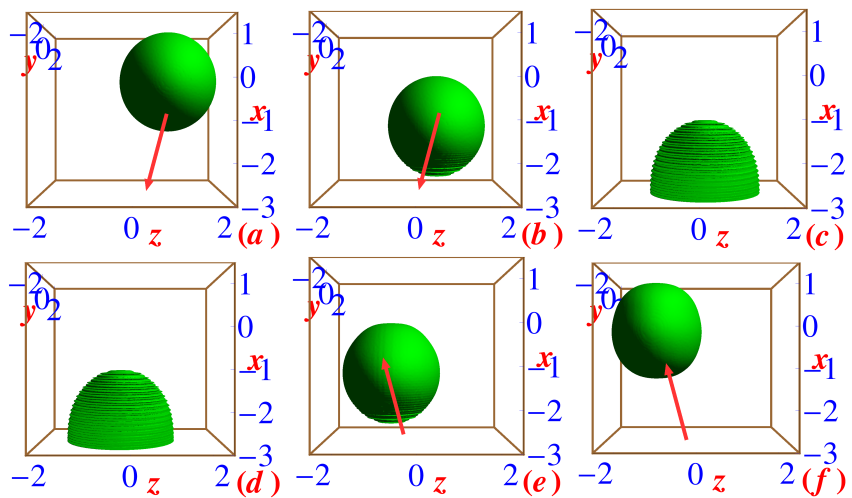

FIG. 9: (Color online) Bouncing off a rigid wall at $x=-3 \mu \mathrm{m}$ of a ${ }^{7} \mathrm{Li}$ quantum ball, with $N=1500, K_{3}=3 \times 10^{-37}(1-i)$ $\mathrm{m}^{6} / \mathrm{s}$, placed at $x=0, z=1 \mu \mathrm{m}$ at $t=0$ and set into motion towards $x=-3 \mu \mathrm{m}, z=0$ with velocity $18.9 \mathrm{~cm} / \mathrm{s}$, illustrated by dimensionless isodensity contours at times (a) $t=0$, (b) $=0.00669 \mathrm{~ms},(\mathrm{c})=0.0134 \mathrm{~ms},(\mathrm{~d})=0.020 \mathrm{~ms},(\mathrm{e})=0.0267$ $\mathrm{ms},(\mathrm{f})=0.0334 \mathrm{~ms}$. The density on the contour is $10^{10}$ atoms $/ \mathrm{cm}^{3}$. The directions of motion of the quantum ball are shown by arrows.

is achieved by just imposing reflecting boundary condition at the surface in the Crank-Nicolson algorithm 22]. The interaction dynamics in this case is illustrated by successive snapshots of isodensity contour before, during and after interaction with the rigid plane in Figs. 8(a)(f). The quantum ball moves without any deformation in Figs. 8(a)-(b), gets deformed in proximation of the rigid plane in Figs. 8(c)-(d), bounces off without any deformation and without any change of speed and with the direction of motion reversed in Figs. 8(e)-(f). The dynamics of the quantum ball is the same as that of an elastic classical ball except near the rigid plane when the quantum ball gets deformed.

The interaction of a quantum ball upon angular impact with a rigid plane is studied next. A quantum ball of $N=1500$ and $K_{3}=3 \times 10^{-37}(1-i) \mathrm{m}^{6} / \mathrm{s}$ is placed at $x=y=0, z=1 \mu \mathrm{m}$ and set into motion towards $x=-3$ $\mu \mathrm{m}, y=z=0$ by multiplying the imaginary-time wave function by $\exp (-i 20 x+5.8 i z)$ to set the quantum ball in motion with an initial speed of $18.9 \mathrm{~cm} / \mathrm{s}$ and performing real-time simulation with this wave function in a box of dimension $240 \times 240 \times 320$. The reflection of the ball from the wall is achieved by imposing reflecting boundary condition at the surface in the Crank-Nicolson algorithm [22]. The uniform motion of the quantum ball without deformation before and after the collision are shown in Figs. 9(a)-(b) and 9(e)-(f), respectively, while its deformation in the proximity of the rigid plane is shown in Figs. 9(c)-(d). The quantum ball bounces like an elastic classical ball with the same speed obeying the classical law of reflection. The results presented so far demonstrate beyond doubt that for large velocities the quantum ball interacts elastically with another quantum ball or an external rigid plane with the conservation of ki-
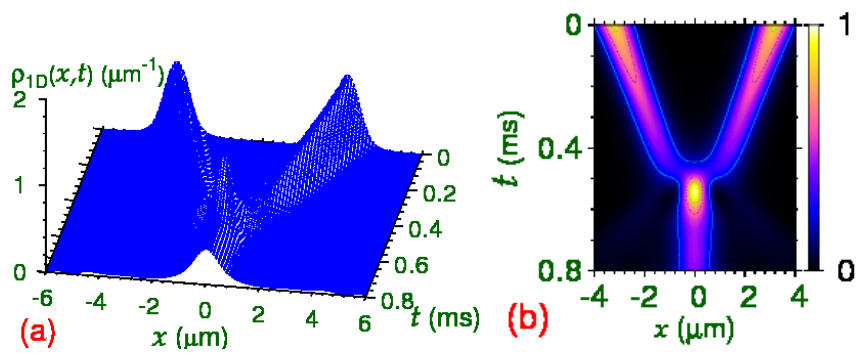

FIG. 10: (Color online) (a) The 1D density $\rho_{1 D}(x, t)$ and (b) its contour plot during the collision of two ${ }^{7} \mathrm{Li}$ quantum balls, with $N=1500$ and $K_{3}=3 \times 10^{-37}(1-0.05 i) \mathrm{m}^{6} / \mathrm{s}$ each, initially placed at $x= \pm 3.2 \mu \mathrm{m}$ at $t=0$ and set into motion towards each other with a velocity $0.45 \mathrm{~cm} / \mathrm{s}$, upon real-time propagation.

netic energy. However, the collision is inelastic at small velocities and large deformation of the quantum ball is possible with the nonconservation of kinetic energy. We performed simulation at small velocities (about one tenth of the velocities considered so far) for the interaction with a rigid plane. We find that the quantum ball bounces off the surface at such low incident velocities but with some deformation in shape and nonconservation of kinetic energy resulting in a reduction in the final velocity.

To study the inelastic collision dynamics we consider two quantum balls each with $N=1500$ and a three-body term with dissipation, $K_{3}=3 \times 10^{-37}(1-0.05 i) \mathrm{m}^{6} / \mathrm{s}$, place them at $x= \pm 3.2 \mu \mathrm{m}, y=z=0$ and set them in motion in opposite directions along the $x$ axis with a small velocity: $v=0.45 \mathrm{~cm} / \mathrm{s}$. In this case we have used a smaller dissipative term to avoid a large loss of atoms over long-time collision dynamics for a small velocity. The dynamics is started by multiplying the respective imaginary-time wave functions by $\exp ( \pm i 0.5 x)$ and performing real-time simulation for the study of dynamics. The imaginary and real-time simulations were performed in boxes of size $256 \times 256 \times 256$ and $512 \times 256 \times 256$, respectively. The dynamics is illustrated by a plot of the time evolution of $1 \mathrm{D}$ density $\rho_{1 D}(x, t)$ in Fig. 10 (a) and the corresponding two-dimensional contour plot is shown in Fig. 10 (b). The two quantum balls come close to each other at $x=0$ and coalesce to form a quantum-ball breather and never separate again. The combined bound system remain at rest at $x=0$ continuing small breathing oscillation because of a small amount of liberated kinetic energy which creates the quantum-ball breather. Hence at sufficiently small incident velocities the collision of two quantum balls lead to the formation of a quantum-ball breather and at large velocities one has the quasi-elastic collision of two quantum balls. 


\section{SUMMARY}

We demonstrated the creation of a stable, stationary BEC quantum ball (a self-bound BEC) under attractive two-body and repulsive three-body contact interactions employing a variational and full numerical solution of the 3D GP equation. The statical properties of the quantum ball are studied by the variational approximation and a numerical imaginary-time solution of the 3D GP equation. The dynamical properties are studied by a realtime solution of the GP equation including an absorptive three-body term. The quantum ball can move with a constant velocity without deformation. At large velocities, the collision between the two quantum balls is quasi elastic with no visible deformation of the final quantum balls. We studied head-on and angular collisions of two quantum balls. In all cases, unlike classical balls, the balls come out of the collision region without deformation maintaining their velocities and directions of motion unchanged. In elastic collision of two classical balls one can have a change in the direction of their motion subject to energy and momentum conservation. At small velocities, the collision between two quantum balls is inelastic with the formation of a quantum-ball breather after collision.

As collapse is not allowed, even in the presence of a very small three-body repulsion, the present suggestion of realizing a trapless BEC quantum ball seems to be attractive from an experimental point of view. The size of a trapped dipolar BEC is determined by the harmonic oscillator lengths of the trap, whereas the size of the present quantum ball is determined by the two-body and threebody interactions. One should start with a tapped dipolar BEC for $N<N_{\text {crit }}$ where no droplet can be formed, viz. Fig. 1(c). Now using the Feshbach resonance technique, one should make the scattering length more attractive to enter the stable domain from unstable domain in Fig. 11(c). If the harmonic trap is weak then initial size of the trapped BEC should be large, and by increasing the two-body attraction the size of the quantum ball could be made much smaller: the sudden change in size will identify the trapless quantum ball.

\section{Acknowledgments}

We thank the Fundação de Amparo à Pesquisa do Estado de São Paulo (Brazil) (Project: 2012/00451-0 and the Conselho Nacional de Desenvolvimento Científico e Tecnológico (Brazil) (Project: 303280/2014-0) for support.
[1] M. H. Anderson et al., Science 269, 198 (1995); K. B. Davis, M. O. Mewes, M. R. Andrews, N. J. vanDruten, D. S. Durfee, D. M. Kurn, and W. Ketterle, Phys. Rev. Lett. 75, 3969 (1995); C. C. Bradley, C. A. Sackett, J. J. Tollett, and R. G. Hulet, Phys. Rev. Lett. 75, 1687 (1995).

[2] F. Dalfovo, S. Giorgini, L. P. Pitaevskii, and S. Stringari, Rev. Mod. Phys. 71, 463 (1999).

[3] M. Greiner, O. Mandel, T. Esslinger, T. W. Hänsch, and I. Bloch, Nature (London) 415, 39 (2002).

[4] E. A. Donley, N. R. Claussen, S. L. Cornish, J. L. Roberts, E. A. Cornell, C. E. Wieman, Nature (London) 412, 295 (2001); S. K. Adhikari, Phys. Rev. A 66, 013611 (2002).

[5] L. Deng, E. W. Hagley, J. Wen, M. Trippenbach, Y. Band, P. S. Julienne, J. E. Simsarian, K. Helmerson, S. L. Rolston, and W. D. Phillips, Nature (London) 398, 218 (1999).

[6] J. R. Abo-Shaeer, C. Raman, J. M. Vogels, and W. Ketterle, Science 292, 476 (2001).

[7] M. R. Andrews, C. G. Townsend, H.-J. Miesner, D. S. Durfee, D. M. Kurn, W. Ketterle, Science 275, 637 (1997).

[8] B. D. Josephson, Phys. Lett. 1, 251 (1962); F. S. Cataliotti, S. Burger, C. Fort, P. Maddaloni, F. Mi- nardi, A. Trombettoni, A. Smerzi, M. Inguscio, Science 293, 843 (2001); S. K. Adhikari, Phys. Rev. A 72, 013619 (2005); Eur. Phys. J. D 25, 161 (2003).

[9] P. W. Anderson, Phys. Rev. 109, 1492 (1958); J. Billy, V. Josse, Z. Zuo, A. Bernard, B. Hambrecht, P. Lu- gan1, D. Clément, L. Sanchez-Palencia, P. Bouyer, and A. Aspect, Nature (London) 453, 891 (2008); G. Roati, C. D'Errico1, L. Fallani1, M. Fattori, C. Fort, M. Zaccanti, G. Modugno, M. Modugno, and M. Inguscio, Nature (London) 453, 895 (2008); S. K. Adhikari and L. Salasnich, Phys. Rev. A 80, 023606 (2009).

[10] V. M. Perez-Garcia, H. Michinel, and H. Herrero, Phys. Rev. A 57, 3837 (1998); F. K. Abdullaev, A. Gammal, A. M. Kamchatnov, and L. Tomio, Int. J. Mod. Phys. B 19, 3415 (2005); K. E. Strecker, G. B. Partridge, A. G. Truscott, and R. G. Hulet, Nature (London) 417, 150 (2002); L. Khaykovich, F. Schreck, G. Ferrari, T. Bourdel, J. Cubizolles, L. D. Carr, Y. Castin, and C. Salomon, Science 256, 1290 (2002).

[11] P. Pedri and L. Santos, Phys. Rev. Lett. 95, 200404 (2005).

[12] Y. S. Kivshar and G. Agrawal, Optical Solitons: From Fibers to Photonic Crystals, (Academic Press, San Diego, 2003).

[13] Y. S. Kivshar and B. A. Malomed, Rev. Mod. Phys. 61, 763 (1989).

[14] S. K. Adhikari, Phys. Rev. A 69, 063613 (2004), Phys. Rev. E 70, 036608 (2004); H. Saito and M. Ueda, Phys. Rev. Lett. 90, 040403 (2003); F. K. Abdullaev, J. G. Caputo, R. A. Kraenkel, and B. A. Malomed, Phys. Rev. A 67, 013605 (2003).

[15] F. Maucher, N. Henkel, M. Saffman, W. Krolikowski, S. Skupin, and T. Pohl, Phys. Rev. Lett. 106, 170401 (2011).

[16] A. Parola, L. Salasnich, and L. Reatto, Phys. Rev. A 57, 
3180(R) (1998); arxiv:9807.390; L. Salasnich, Phys. Rev. A 61, 015601(1999).

[17] Z. Shotan, O. Machtey, S. Kokkelmans, and L. Khaykovich, Phys. Rev. Lett. 113, 053202 (2014).

[18] S. K. Adhikari, Laser Phys. Lett. 14, 025501 (2017).

[19] V. M. Perez-Garcia, H. Michinel, J. I. Cirac, M. Lewenstein, and P. Zoller, Phys. Rev. Lett. 77, 5320 (1996).

[20] R. Y. Chiao, E. Garmire, and C. H. Townes, Phys. Rev. Lett. 13, 479 (1964).

[21] E. R. I. Abraham, W. I. McAlexander, J. M. Gerton, R. G. Hulet, R. Côté, and A. Dalgarno, Phys. Rev. A 55, R3299 (1997).

[22] P. Muruganandam and S. K. Adhikari, Comput. Phys. Commun. 180, 1888 (2009); D. Vudragović, I. Vidanović, A. Balaž, P. Muruganandam, and S. K. Adhikari, Comput. Phys. Commun. 183, 2021 (2012).
[23] P. Muruganandam and S. K. Adhikari, J. Phys. B 36, 2501 (2003).

[24] B. Satarić, V. Slavnić, A. Belić, A. Balaž, P. Muruganandam, and S. K. Adhikari, Comput. Phys. Commun. 200, 411 (2016); V. Lončar, A. Balaž, A. Bogojević, S. Skrbić, P. Muruganandam, and S. K. Adhikari, Comput. Phys. Commun. 200, 406 (2016); R. Kishor Kumar, L. E. Young-S., D. Vudragović, A. Balaž, P. Muruganandam, and S. K. Adhikari, Comput. Phys. Commun. 195, 117 (2015); V. Lončar, L. E. Young-S., S. Škrbić, P. Muruganandam, S. K. Adhikari, and A. Balaž, Comput. Phys. Commun. 209, 190 (2016); L. E. Young-S., D. Vudragovic, P. Muruganandam, S. K. Adhikari, A. Balaz, Comput. Phys. Commun. 204, 209 (2016). 